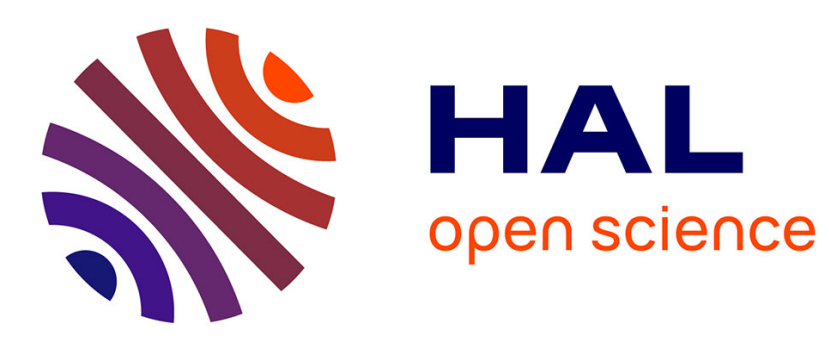

\title{
History, rare and multiple events of mechanical unfolding of repeat proteins
}

Fidan Sumbul, Arin Marchesi, Felix Rico

\section{To cite this version:}

Fidan Sumbul, Arin Marchesi, Felix Rico. History, rare and multiple events of mechanical unfolding of repeat proteins. Journal of Chemical Physics, 2018, 148 (12), 10.1063/1.5013259 . hal-01792492

\section{HAL Id: hal-01792492 \\ https://hal-amu.archives-ouvertes.fr/hal-01792492}

Submitted on 15 May 2018

HAL is a multi-disciplinary open access archive for the deposit and dissemination of scientific research documents, whether they are published or not. The documents may come from teaching and research institutions in France or abroad, or from public or private research centers.
L'archive ouverte pluridisciplinaire HAL, est destinée au dépôt et à la diffusion de documents scientifiques de niveau recherche, publiés ou non, émanant des établissements d'enseignement et de recherche français ou étrangers, des laboratoires publics ou privés. 


\title{
. History, rare and multiple events of mechanical unfolding of repeat proteins
}

\author{
Fidan Sumbul, Arin Marchesi, Felix Rico* \\ U1006, Aix-Marseille Université \& INSERM, 163 avenue de Luminy, 13009 Marseille,
} France.

\begin{abstract}
Mechanical unfolding of proteins consisting of repeat domains is an excellent tool to obtain large statistics. Force spectroscopy experiments using AFM on proteins presenting multiple domains have revealed that unfolding forces depend on the number of folded domains (history) and have reported intermediate states and rare events. However, the common use of unspecific attachment approaches to pull the protein of interest holds important limitations to study unfolding history and may lead to discarding rare and multiple probing events due to the presence of unspecific adhesion and uncertainty on the pulling site. Site-specific methods recently emerged minimize this uncertainty and would be excellent tools to probe unfolding history and rare events. However, detail characterization of these approaches is required to identify their advantages and limitations. Here, we characterize a site-specific binding approach based on the ultrastable complex dockerin/cohesin III revealing its advantages and limitations to assess the unfolding history and to investigate rare and multiple events during the unfolding of repeated domains. We show that this approach is more robust, reproducible and provide larger statistics than conventional unspecific methods. We show that the method is optimal to reveal the history of unfolding from the very first domain, and to detect rare events, while being more limited to assess intermediate states. Finally, we quantify the unfolding forces required to unfold two molecules pulled in parallel, difficult using unspecific approaches. The proposed method represents a step forward towards more reproducible measurements to probe protein unfolding history and opens the door to systematic probing of rare and multiple molecule unfolding mechanisms.
\end{abstract}

\section{Introduction}

Force spectroscopy experiments using nanotools provide unique insights of single molecule mechanics ${ }^{1-3}$. One of the early atomic force microscopy (AFM) experiments on single molecules reported the unfolding of the muscle protein titin, which revealed a sawtooth-like force pattern reflecting the sequential unfolding of individual protein domains ${ }^{4}$. Since then, our understanding of the response of individual proteins to force has progressed considerably. 
The current picture describes protein unfolding through a rough energy landscape governed by thermal fluctuations and presenting, sometimes, multiple barriers and/or reaction pathways 5-8. To obtain large statistics and to isolate the forced unfolding behaviour of a particular protein domain, a useful approach involves the construction of a concatamer of the repeated protein domains where multiple copies of the same domain are linked in series. Robust analysis of protein unfolding requires proper orientation of the protein of interest, difficult using the common unspecific pulling approaches. While analyzing the measurements on repeated protein domains, the measured unfolding forces are commonly pooled together independently of the number of pulled domains (history), assuming identical behaviour ${ }^{4,9,10}$. However, single molecule experiments have revealed the effect of force history in the mechanical response ${ }^{11-15}$. Indeed, as predicted theoretically and observed in experiments, the unfolding forces of concatamers made of multiple repeats of identical protein domains have shown to depend on the number of remaining folded domains, thus on their history, and on the number of repeated domains in the construct ${ }^{16-19}$. However, unspecific binding is known to obscure the first unfolding peaks, thus compromising the accuracy of the observed results ${ }^{14}$. Moreover, using unspecific attachement, the molecule is pulled from an unknown position along its length. Thus, the number of folded domains being pulled is often unknown ${ }^{15}$.

Force spectroscopy measurements on single proteins allow the observation of intermediate states and rare events, often hidden in ensemble average bulk methods ${ }^{20}{ }^{21}$. However, these events may be discarded if not expected a priori and have been rarely reported as their detection requires probing the same molecule repeated times and/or pulling traces without unspecific force peaks. In addition, events reflecting parallel unfolding of multiple proteins have been commonly discarded, likely due to the difficult interpretation of the results when using unspecific binding approaches. Thus, proper exploration of protein mechanics requires a robust approach minimizing the presence of unspecific events, knowledge of the exact number of folded domains being pulled and a clear fingerprint for the selection of successful events that would not rely on the expected unfolding patterns of the protein of interest (POI). While the use of covalent attachment of biomolecules to tip and sample is routine for unbinding or recognition experiments of receptor-ligand interactions since the early beginning 22, 23 , probing proteins using unspecific binding has been the norm in unfolding experiments ${ }^{4}$, 5, 9 . The major issue of unspecific binding is that unfolding patterns not corresponding to the expected fingerprint, e.g. not regular spacing between peaks, may be systematically discarded. This may lead to biased results and to the rejection of traces presenting rare events and/or 
unexpected intermediate states ${ }^{14}$. Using a reproducible attachment to pull from a specific site on the biomolecule of interest would avoid this problem.

Recently, various methods have been developed involving covalent or ultrastable bonds between the probe and the POI ${ }^{24-29}$. The common methods used in optical tweezers experiments based on DNA handles attached to the beads using biotin/(strept)avidin and digoxigenin/anti-digoxigenin specific binding are in general not applicable to most AFM measurements of protein unfolding due to the high forces involved ${ }^{30}$. Thus, other strategies have been developed that to allow stronger site-specific attachment. Covalent bonds formed by peptide tags have been successfully applied to unfold proteins using AFM. A recent development used a peptide (SpyTag) forming an amide bond to its protein partner (SpyCatcher) in minutes ${ }^{26}$. The bond was shown to sustain large forces, (in the $\mathrm{nN}$ range) before breaking, being thus suitable to unfold almost any protein domain. A main drawback of this method is that the complex requires long interaction times $\sim 20 \mathrm{~s}$, which led to important unspecific forces. To minimize this unspecific interaction forces, the authors used agarose beads to which attach the protein of interest (POI) ${ }^{26,31}$. Despite its low unspecific binding, agarose supports provide a compliant surface and is limited in terms of available bioconjugation methods. An alternative approach was later developed based on HaloTag, a Haloalkane Dehalogenase forming an ester bond with a chloroalkane-functionalized surface. Being covalent, the formed bond also sustains $\mathrm{nN}$ forces over long periods of time, and has been successfully applied to unfold proteins using AFM ${ }^{25,32}$. While one end of the POI was immobilized using the HaloTag approach, the other end required thiol binding to a gold substrate, which limits its applicability to proteins without cysteine residues and introduces strong optical interference artifacts. Both SpyTag and HaloTag methods form covalent bonds, not reversible, which may compromise the lifetime of the functionalized tip, since any covalent bond along the pulled chain may disrupt.

More recently, a strategy developed by the Perkins group used site-specific attachment using click chemistry on one end and a biotin/streptavidin bond for pulling from the POI ${ }^{33}$. This approach was successfully applied to unfold three different proteins (calmodulin, rubredoxin and the artificial triple-helical $\alpha_{3} \mathrm{D}$ ). Compared to common unspecific approaches, the method provides orders of magnitude higher binding efficiency, being thus more robust and much faster in providing large statistics. Moreover, unlike covalent attachment, the method has the advantage of forming a reversible, site-specific binding. However, its application is limited to labile proteins (unfolding at forces of a few tens of $\mathrm{pN}$ ), due to the relatively low binding strength of the streptavidin/biotin complex used to pull from the POI. 
One of the most versatile approaches uses the cellulosome ultrastable complex formed by dockerin and cohesin III domains ${ }^{34}$. The approach proposed by Gaub and coworkers makes use of site-specific covalent attachment of one end using a ybbR tag (a sequence of 11 amino acids), and an ultrastable but reversible receptor/ligand bond (Cohesin/Dockerin III) to grab the POI ${ }^{27-29,35,36}$. In practice, a DNA construct is engineered to include the ybbR tag, the POI and type III dockerin concatenated. The expressed fusion protein is then covalently immobilized via the ybbR tag to the sample surface leaving the free dockerin III end to the bulk, while cohesin III is covalently attached to the tip ${ }^{27,28}$. This provides the two main advantages: site specific linkage and reversible attachment, with the additional benefit of the high binding strength of the dockerin/cohesin III complex, which unbinds at forces above $300 \mathrm{pN}$ at conventional pulling velocities, higher than the forces required to unfold most of the proteins probed so far ${ }^{37}$. Moreover, it provides a characteristic unfolding fingerprint that further allows identification of specific unfolding events. In addition, it is suitable for cell-free expression systems, making it even more versatile. Therefore, the dockerin/cohesin III strategy assures specificity, proper orientation, reversibility and reproducibility, while avoiding the use of highly reflecting surfaces, such as gold, and minimizing unspecific binding. The dockerin/cohesin III approach seems, thus, to be an excellent option for force spectroscopy measurements in search of rare events, intermediate states and history dependence. However, little data has been provided about the robustness, binding efficiency and the versatility of this approach compared to conventional methods. While site-specific approaches emerge, detailed characterization of the different methods is required to assess their advantages and limitations to allow the best choice for the specific question to answer. Here, we assess this latter method, showing its advantages and limitations and confronting it with unspecific attachment approaches.

We designed a recombinant protein presenting eight repeated domains of cardiac titin I91 fused with type III dockerin. Site-specific immobilization on the sample surface and cantilevers coated with cohesin III allowed control of the probing position and reproducible evaluation of the mechanical unfolding. We first compare the success rate of valid unfolding traces using conventional unspecific attachment and the dockerin/cohesin III approach, showing that the latter is more reproducible and leads to larger statistics. We then show its versatility by assessing the effect of unfolding history, unambiguously assessing all unfolding peaks, not possible with unspecific approaches. Finally, we investigate rare events and, for the first time, quantify the forces required to unfold two molecules pulled simultaneously. 


\section{Materials and Methods}

\section{Titin concatamer chimera construction and purification}

The cDNA sequence coding for the I91 titin repeat was modified to insert the restriction sites BamHI at the 5' end and BgIII at the 3' end. The gene was then cloned into the pET28a plasmid which has been modified to accommodate a His $10^{- \text {tag }}$ between NcoI and BamHI restriction sites. The 8-domain synthetic gene was then constructed by iterative cloning of monomer into monomer, dimer into dimer, and tetramer into tetramer ${ }^{9}$. The DNA sequence corresponding to the titin octamer repeats $\left(\mathrm{T}_{8}\right)$ was confirmed by Sanger sequencing. ybbRtag ${ }^{35,38}$ was introduced by oligo annealing and cloning into the $\mathrm{pET} 28 \mathrm{a}-\mathrm{T}_{8}$ vector which was linearized with BamHI. The DNA fragment coding for XMod and dockerin-III was then cloned into the KpnI and XholI restriction sites. The DNA sequence encoding for $y b b R, \mathrm{~T}_{8}$ and XMod-dockerin-III (from N- to C-terminus) domains was confirmed by Sanger sequencing. The chimeric pET28a expression plasmid was transformed into Escherichia Coli BL21 (DE3) competent cells. Transfected cells were grown at $37^{\circ} \mathrm{C}$ in $1 \mathrm{~L}$ Luria-Bertani media with kanamycin $(50 \mathrm{ng} / \mathrm{ml})$ until the cell density (OD600) reached 0.6. To induce protein expression, IPTG (Formedium) was added to a final concentration of $0.3 \mathrm{mM}$ and the medium was incubated at $20^{\circ} \mathrm{C}$ overnight. After harvesting, cells were resuspended and lysed using probe sonication in $5 \mathrm{ml}$ buffer $\mathrm{B}\left(100 \mathrm{mM} \mathrm{NaCl}, 2 \mathrm{mM} \mathrm{CaCl}_{2}, 20 \mathrm{mM}\right.$ HEPES-NaOH, $\mathrm{pH}$ 7.6) with $1 \mathrm{mM}$ PMSF protease inhibitor (Sigma). titin chimera was solubilized in $0.1 \%$ Triton X-100 (Sigma) and $10 \mu \mathrm{g} / \mathrm{mL}$ DNaseI (Roche) by gentle shaking for 30 minutes at $4^{\circ} \mathrm{C}$. The resulting crude cell extract was centrifuged at $10,000 \mathrm{~g}$ for 45 minutes at $4^{\circ} \mathrm{C}$ and the supernatant fluid was applied to Ni-NTA affinity resin (Generon) which was preequilibrated in buffer B. The resin was then washed three times with buffer B containing 50 $\mathrm{mM}$ imidiazole to remove non-specifically bound proteins, and finally eluted with $250 \mathrm{mM}$ imidiazole. After elution, the solution was dialyzed overnight at $4^{\circ} \mathrm{C}$ to remove the imidazole. Titin octamers presenting two cysteines at the N-terminus to attach to gold-coated surfaces were purified as described before ${ }^{9,10}$.

\subsection{Surface and tip functionalization}

For the specific binding approach, we used eight concatenated titin repeats followed by XMod and dockerin-III domains on the sample surface, while CBM and cohesin-III on the cantilever (Fig. 1). The dockerin and cohesin III domains were at the free end of the respective construct while the other domain was covalently attached to the surface. For the specific dockerin/cohesin III approach, glass surfaces $(12 \mathrm{~mm}$ diameter coverslips, VWR 
International) and silicon nitride cantilevers (BL-AC40TS-C2, Olympus and MLCT, Bruker) were first rinsed with acetone and then cleaned for 5 minutes with oxygen plasma (Plasma cleaner Zepto, Diener electronic) under conditions of $80 \mathrm{~W}$ power at 0.6 mbar. The cleaned coverslips and cantilevers were rinsed in analytical grade ethanol (more than $99.9 \%$ purity, Sigma), and silanized with 5\% (3-aminopropyl)-dimethyl-ethoxysilane (APDMES, abcr $\mathrm{GmbH}$ ) in ethanol for 10 minutes at room temperature. The silanized glass surfaces and cantilevers were then rinsed with ethanol and then baked at $80^{\circ} \mathrm{C}$ for 1 hour. In order to deprotonate the amino-groups on the surface of coverslips and cantilevers to allow amidebond formation with the NHS-ester group of the linker, the cover slips and cantilevers were immersed immediately in PBS pH:9 and incubated overnight at $+4^{\circ} \mathrm{C}$. The deprotonated amino functionalized coverslips and cantilevers were then incubated in 5mM NHS-PEG(27)Maleimide (Polypure) in phosphate-buffered saline (PBS: $10 \mathrm{mM} \mathrm{Na} \mathrm{HPO}_{4}, 1.76 \mathrm{mM}$ $\left.\mathrm{KH}_{2} \mathrm{PO}_{4}, 137 \mathrm{mM} \mathrm{NaCl}, 2.7 \mathrm{mM} \mathrm{KCl}, \mathrm{pH}: 7.2\right)$ for 1 hour at room temperature. The PEGylated glass surfaces and cantilevers were rinsed with milli-Q water and then incubated in $20 \mathrm{mM}$ coenzyme A-trilithium salt (Sigma) in 50mM Na $2 \mathrm{HPO}_{4}, 50 \mathrm{mM} \mathrm{NaCl}, 10 \mathrm{mM}$ EDTA pH 7.2 for 1 hour at room temperature. After rinsing with Milli-Q water, $\sim 300 \mu \mathrm{g} / \mathrm{mL}$ ybbR-T ${ }_{8}$ XMod-dockerin was added on the treated side of the glass surfaces in presence of $1 \mu \mathrm{M}$ Sfp in PBS pH 7.2 buffer with $20 \mathrm{mM} \mathrm{MgCl}_{2}$ and incubated for 1 hour at room temperature, enabling covalent immobilization of multi protein construct via Sfp-catalyzed ligation of coenzyme A and the ybbR tags. The cantilevers were functionalized by incubating with $100 \mu \mathrm{g} / \mathrm{mL}$ ybbRCohesin III in presence of $1 \mu \mathrm{M}$ of Sfp in PBS pH 7.2 buffer and $20 \mathrm{mM} \mathrm{MgCl}_{2}$ for 1 hour at room temperature. The functionalized coverslips and cantilevers were rinsed with PBS pH 7.2 buffer to remove the unbound or loosely bound proteins. The cantilevers and surfaces were stored in PBS pH 7.2 buffer until the measurements.

For the unspecific binding approach, gold-coated glass coverslips $(6 \mathrm{~mm}, 5 \mathrm{~nm}$ of gold on $3.5 \mathrm{~nm}$ of chromium) were cleaned for 5 minutes with oxygen plasma under conditions $80 \mathrm{~W}$ power and $0.6 \mathrm{mbar}$. Then, a drop of $\sim 300 \mu \mathrm{g} / \mathrm{mL}$ of $\mathrm{T}_{8}$ protein presenting two cysteines at the N-terminus was incubated in PBS pH 7.2 for 1 hour at room temperature. Finally, the surfaces were rinsed with PBS pH 7.2 to remove the unbound or loosely bound proteins and stored in PBS pH 7.2 until measurements.

\subsection{Single molecule force spectroscopy (SMFS) measurements}

For the force measurements, a commercial AFM setup (Nanowizard 3, JPK) was used. The deflection sensitivity, resonance frequency, quality factor and spring constant of the 
cantilevers were determined prior to each measurement. The spring constant of the cantilevers was determined using Sader method with the thermal spectrum in air ${ }^{39,40}$. The sensitivity of the photodiode was extracted from the thermal noise spectrum in liquid ${ }^{41-44}$. Measurements were performed in PBS $\mathrm{pH}$ 7.2. Force-extension traces were acquired at a velocity of $\sim 1.5 \mu \mathrm{m} / \mathrm{s}$ with $100 \mathrm{~ms}$ contact time at $\sim 250 \mathrm{pN}$ contact force. To compare the two attachment procedures, force measurements were performed over three different areas on the sample surface of $10 \mu \mathrm{m}$ side. Areas were divided into 10x10 points and three measurements were collected at each point, leading to a total of 900 number of force-extension traces.

\subsection{Data processing}

Dockerin III specifically binds to cohesin III and the complex unbinds at forces above $300 \mathrm{pN}$ at conventional pulling rates. This specific interaction also provides a signature-unfolding fingerprint that further allows identification of specific unfolding events. This ensures unfolding of the desired protein assuring specificity, proper orientation, reversibility and reproducibility. Therefore, only the force curves with a dockerin/cohesin III unbinding were taken into account for further analysis. The specific unfolding event were detected using an in-house built semiautomatic procedure developed in Matlab environment (Mathworks Inc.) and the unfolded chain length (contour length) of each unfolded domain was determined using worm-like chain (WLC) model (Eq. 3, below).

\subsection{Monte Carlo simulations}

To simulate the unfolding of the 8 titin I91 domains, we used the elastically coupled two-level model described by Rief and coworkers but considering a distribution of barrier heights and distances to the transition state ${ }^{45}$. For simplicity, we used the transition rate described by Bell

$$
k^{0}=\omega e^{-\Delta G^{*} / k_{B} T} \quad 1 .
$$

where $\omega$ is the attempt frequency, $\Delta G^{*}$, the activation barrier for unfolding, $k_{B}$, the Boltzmann constant and $T$, the temperature. In the linear approximation developed by Bell, the unfolding rate will increase exponentially with an applied force $(F)$

$$
k(F)=\omega e^{-\frac{\Delta G^{*}-F x_{u}}{k_{B} T}}=k^{0} e^{F x_{u} / k_{B} T} \quad 2 .
$$

being $x_{u}$, the distance to the activation barrier.

The stretching regime was modelled using the worm-like chain (WLC) model for the force response upon extension $(x)$

$$
F(x)=\frac{k_{B} T}{p}\left(\frac{1}{4\left(1-\frac{x}{L}\right)^{2}}-\frac{1}{4}+\frac{x}{L}\right) 3
$$


where $p$ and $L$ are the persistence and contour lengths, respectively.

The simulation started by extending the polymer with an initial contour length $\left(L_{0}=160 \mathrm{~nm}\right.$, experimentally determined length, including the CBM domain and the PEG linkers) at a velocity $v_{c}$ starting from $x=0$. We assumed the cantilever being in series with the worm like chain to calculate numerically the extension $(\Delta x)$ per time interval $(\Delta t)$ as

$$
\Delta x=v_{c} \Delta t \quad 4 .
$$

With this additional extension, the force was calculated from Eq. 3 and the corresponding transition rate from Eq. 2. The probability of unfolding any of the $N_{\mathrm{f}}$ folded domains was calculated for each time interval as

$$
d P_{u}=\sum_{i} k_{i}(F) \Delta t \quad 5 .
$$

being $k_{\mathrm{i}}(F)$, the unfolding rate of each domain, which would be slightly different due to the imposed dynamic disorder. $d P_{u}$ was then compared with a generated random number. If the probability of one of the domains was higher than the generated random number, the domain was assumed to unfold, and the total contour length was changed by adding the contour length of an unfolded domain ( $28 \mathrm{~nm}$, determined experimentally). The force was then recalculated and pulling continued until all domains unfolded, $N_{\mathrm{f}}=0$.

\subsection{Data analysis}

Unfolding force histograms were modelled using the Bell-Evans model for the probability density distribution

$$
p(F)=\frac{k^{0}}{r_{f}} \exp \left\{\frac{F}{k_{B} T x_{u}}\right\} \exp \left\{\frac{k^{0}}{k_{B} T x_{u} r_{f}}\left[1-\exp \left(\frac{F}{k_{B} T x_{u}}\right)\right]\right\} \quad 6 .
$$

being $r_{\mathrm{f}}$, the loading rate. When $n$ domains are being pulled, the constant $k^{0}$ is modified to become $k^{0} n !^{1 / n} 19$.

\section{Results and Discussion}

We carried out single molecule force spectroscopy measurements using AFM by grabbing the molecules with the tip employing unspecific and specific approaches. In the unspecific attachment method, the concatenated eight repeats of titin $\mathrm{I}_{91} \mathrm{~T}_{8}$ construct was attached to the gold-coated surface via two cysteines present in the N-terminus, and then probed with a bare silicon nitride AFM tip, which grabbed the molecule unspecifically at a random position along its length. In the specific attachment method, the eight repeats of titin 191 construct featured a ybbR tag at the N-terminus and the dockerin III domain at the C-terminus (ybbR$\mathrm{T}_{8}$-dockerin) and was covalently immobilized to the glass surface through the ybbR-tag via 
Sfp-catalyzed ligation, with the free end exposed dockerin III to the bulk. Cohesin III was then covalently attached to the tip and used to pull from the dockerin III domain ${ }^{27,28}$. A PEG linker mediated the surface-protein immobilization. The schematic representation of the experimental setup is shown in Fig. 1a. The dockerin/cohesion III complex dissociates at forces $\sim 300 \mathrm{pN}$ at the pulling rate used in the experiment, which is higher than the unfolding forces of titin I91 domains. This rupture forces are considerably lower than those reported before ${ }^{46,47}$, which may be explained by the absence of calcium ions in our measurement buffer since dockerin III contains two calcium binding loops, one in the cohesin III binding region. A typical unfolding trace of the designed polyprotein complex resulted in a saw-tooth like pattern of the unfolding forces starting with the extension of the PEG linkers, the unfolding of the CBM domain (with a contour length of $\sim 65 \mathrm{~nm}$ ) followed by unfolding of the eight repeats of titin 191 domains ( $\sim 28 \mathrm{~nm}$ contour length) and ended with the final dissociation of dockerin/cohesin III complex shown in (Fig. 1b).

We compared the specific and unspecific methods by acquiring force curves across the two types of titin coated surfaces using bare or cohesin III coated tips. Figure 2 shows the success rate obtained with the two alternative attachment methods using two different types of cantilevers (AC40 and MLCT-E). To calculate these probabilities, we only considered as

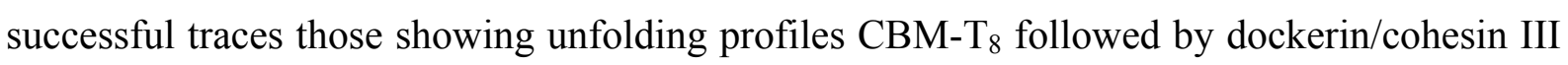
unbinding, for the specific method. For the unspecific approach, considering only the very few events showing 8 titin unfolding peaks would have not allowed a significant statistical analysis. Thus, we were less conservative and considered curves showing 2 unfolding peaks followed by final detachment (this is the minimum required to assure that at least one of the peaks is due to the POI ${ }^{18}$ ). The probability of adhesion refers to the percentage of successful traces out of the total approach/retract cycles collected. Each probability of adhesion was calculated over 900 approach/retract cycles collected at each of three different sample areas, as described in the Methods, and averaged over the different areas. Error bars show standard deviations. The difference between the probabilities is a representative example, while the dispersion is actually higher when comparing tips coated on different days (ranging from $\sim 1 \%$ up to $\sim 30 \%$ using the specific method, and between $\sim 0.01 \%$ up to $\sim 5 \%$ using the unspecific method). The probability of adhesion obtained with specific approach were $15 \%$ and $8 \%$ for AC40 and MLCT-E, respectively, whereas with the bare AC40 and MLCT-E the obtained probabilities were $0.7 \%$ and $1.6 \%$, respectively. The nominal radii of AC40 and MLCT-E tips is considerably different, $8 \mathrm{~nm}$ (maximum $15 \mathrm{~nm}$ ) and $20 \mathrm{~nm} \mathrm{(maximum} 60 \mathrm{~nm}$ ), 
respectively. Thus, MLCT-E tips provide a larger area of contact than AC40. Nonetheless, while important for the unspecific method, specific binding didn't seem to be significantly affected. This is reasonable, while for unspecific attachment a larger contact area provides higher probability of placing the tip along a $\mathrm{T}_{8}$ molecule, in the case of specific attachment the relatively large dimensions of the cohesin III molecule imply that only two or three molecules are present at the very tip apex, thus the molecule mobility and density of tip and sample coating and the specific binding affinity governs the attachment probability. Importantly, the success rate is about one order of magnitude higher using the specific method than with the unspecific one. It thus provides a more reproducible and robust approach for single molecule measurements, resulting in considerably larger statistics and more controlled pulling conditions. Therefore, the specific method offers an excellent approach to study unfolding events of strictly individual domains, taking into account their unfolding history and opening the door to the systematic observation of rare events.

\section{Unfolding history}

As suggested the representative force trace in Fig. 1b, we observed that the average unfolding forces for titin domains depend on the unfolding history, i.e. on the number of unfolded domains (Fig. 3). Indeed, the higher the number of unfolded domains, the higher the force. To reduce the variability in the unfolding history, and to reflect the robustness of the method, even from a limited number of measurements, the data shown in Fig. 3a-c was obtained from a single experimental set of $\sim 900$ force curves leading to about 50 successful events. Previous experiments and theory suggested a biphasic response, unfolding forces being higher for the first unfolding domains, decreasing to a minimum and increasing again for large number of unfolding domains $14,15,18,19$. This biphasic behaviour depended on the number of domains and the cantilever spring constant and was proposed to be governed by the competition between two effects as the number of unfolded domains increases: 1) the probability of unfolding decreases, resulting in an increase in the unfolding forces, 2) the applied loading rate decreases as a response to excess contour length due to already unfolded domains, resulting in a decrease in the unfolding forces. This interpretation seemed to describe successfully the experimental results except for the first unfolding peak ${ }^{14}$. Despite of the careful experimental conditions and the rigour in the peak selection criteria, this discrepancy was explained by the use of unspecific binding approaches, leading to underestimation of the first unfolding peaks due to inclusion of nonspecific protein-protein and domain-surface interactions ${ }^{14,15}$. Previous works on non-identical repeats of titin (e.g. I91-I96 and I98, also 
named repeats I27-I32 and I34) have reported that the larger the number of unfolded domains, the higher the unfolding forces, with no apparent minimum ${ }^{29,48}$. However, when using nonidentical domains, the different mechanical stability of the various domains suggested that weaker domains unfold first. Our data on identical I91 domains show a history behaviour similar to the latter (force increase with the number of unfolded domains), with no apparent minimum but, importantly, with the certainty that non-specific events were discarded, thus with an unambiguous knowledge of the number of domains being pulled for each force trace. In our case, the first titin unfolding occurred at $158 \pm 16 \mathrm{~nm}$ (mean $\pm \mathrm{SD}$ from all processed curves) and increased continuously until the eighth domain, which unfolded at an average force of $182 \pm 19 \mathrm{pN}$. The large length of the probed molecule before titin unfolding explains the absence of biphasic behaviour. Indeed, in our measurements, the first titin unfolding occurred at around $160 \mathrm{~nm}$ (average from all processed curves), due to the extension of the unfolded CBM domain and the PEG linkers. Although previous works do not mention this initial contour length, probably because it is difficult to determine it precisely using unspecific binding approaches, in our system this length is likely larger. At this initial long contour length, the minimum is expected to disappear, because the loading rate does not change importantly with the peak number, and the probability of unfolding is the main contributing mechanism ${ }^{14}$. In effect, our reported history of average unfolding forces was well described by a Monte Carlo (MC) simulation based on the Bell-Evans model with parameters $k^{0}=10^{-}$ ${ }^{3} \mathrm{~s}^{-1}$ and $x_{\mathrm{u}}=0.29 \mathrm{~nm}$. It is important to note that non-Markovian dynamics was not required to describe the unfolding history behaviour, suggesting that, if existent, disorder might have a dynamic origin ${ }^{49,50}$ (Fig. 3b). Previous works using the Bell model are in agreement with these values of the energy landscape parameters ${ }^{9,15}$. As shown in Fig. 3b, except peak 5, all average simulated forces fall within the experimental error. Importantly, a minimum average force at peak 3 was still present if the spring constant of the cantilever in series to the WLC was not considered in the simulation (data not shown).

The average unfolding forces for each peak ranged from $\sim 160 \mathrm{pN}$ to $\sim 180 \mathrm{pN}$, while the average value for all peaks 1-8 pooled together was $166 \mathrm{pN}$ (Fig. 3c). The coefficient of variation $(\mathrm{CoV})$ was similar in both cases, being $9.9 \pm 0.5 \%$ (mean $\pm \mathrm{SD}$, ranging from $9 \%$ to $11 \%$ ) for each peak, while $10.1 \%$ for all the peaks pooled together. The difference in the average unfolding force is remarkable and suggests that averaging all peaks may lead to changes in the determination of the energy landscape parameters, particularly for concatemers with a large number of repeated domains. To assess the induced uncertainty when pooling all peak forces, we fitted the Bell-Evans force probability expression (Eq. 6) to the experimental 
histogram, obtaining $k^{0}=4.4 \times 10^{-3} \mathrm{~s}^{-1}$ and $x_{\mathrm{u}}=0.26 \mathrm{~nm}$, slightly different from the values obtained from the MC simulation $\left(k^{0}=10^{-3} \mathrm{~s}^{-1}\right.$ and $\left.x_{\mathrm{u}}=0.29 \mathrm{~nm}\right)$. The obtained shorter distance to the transition state is reasonable because pooling all unfolding forces leads to slightly wider force histograms. Thus, averaging over different peaks might lead to slight underestimation of $x_{\mathrm{u}}$, while still leading to reasonable values of the energy landscape parameters. Indeed if we compare with the results in the literature, from similar Bell-Evans fits, we observe that this underestimation is well within the experimental dispersion, with values for $x_{\mathrm{u}}$ between $0.25 \mathrm{~nm}$ and $0.36 \mathrm{~nm}$ reported for the very same titin I91 domain $9,15,18$, 51-53. For the dissociation rate, pooling all peaks together results in a higher value than that form the Monte Carlo simulation. $k^{0}$ is more prone to error due to extrapolation and indeed values ranging from $5 \times 10^{-5} \mathrm{~s}^{-1}$ to $2.1 \times 10^{-3} \mathrm{~s}^{-1}$ have been reported. Thus, errors of about one order of magnitude are to be expected for the dissociation rates ${ }^{9,15,51-53}$. This error is of course more important when fitting the probability of unfolding forces at a single pulling velocity and will be reduced when using average forces at different pulling velocities, the wider the dynamic range, the lower the expected error ${ }^{10}$. Since we know the exact number of domains being pulled in each force trace, we can conclude that the larger the number of pulled domains, the shorter the resulting $x_{\mathrm{u}}$ when non pooling all peaks together, due to the wider than expected dispersion of unfolding forces. Indeed, pooling peaks in decreasing order (from all 8 to just 2) leads to $x_{\mathrm{u}}$ fitted values ranging from $0.26 \mathrm{~nm}$ down to $0.33 \mathrm{~nm}$. While we can predict a certain trend, a simple expected result due to peak pooling is difficult to be assessed, since strong correlation between $x_{\mathrm{u}}$ and $k^{0}$ has been shown and reasonable fits are obtained even with $k^{0}$ values changing by an order of magnitude ${ }^{53}$. In Fig. S1 we report the likelihood function $\exp \left(-\chi^{2} / 2\right)$ for the fit with parameters $x_{\mathrm{u}}$ and $k^{0}$ ranging from $0.23 \mathrm{~nm}$ to $0.3 \mathrm{~nm}$ and $10^{-5} \mathrm{~s}^{-1}$ to $10^{-2} \mathrm{~s}^{-1}$, respectively, showing the dependence between the two parameters. This dependence is expected, since the Kramers' definition of $k^{0}$ depends on the curvature of the energy landscape, thus, on $x_{\mathrm{u}}{ }^{54}$. A better fitting variable might be the molecular diffusion constant, which would probably be les prone to error. In spite of this dependence, not considering the unfolding history may be the cause of the reported nonexponential kinetics in force-clamp experiments, due to the slightly different unfolding probabilities of early and late domains ${ }^{55,56}$.

\section{Rare events}

The observation of rare and/or poorly populated events, such as misfolding, double attachment, and intermediate events, has been relatively overlooked in the literature, likely 
because of the limitations of unspecific probing approaches ${ }^{21}$. The built construct and proposed approach allowed us observing a number of rare events that might have been discarded using unspecific approaches. Some of these rare events are summarized in Fig 4. In around $2 \%$ of the curves we observed a pronounced step before a titin unfolding with a contour length of $\sim 12 \mathrm{~nm}$ (trace 1, red arrow, also visible in trace 4, eighth peak). While it could be initially attributed to an intermediate of titin I91, it was likely due to partial unfolding of the dockerin III domain and not to titin unfolding ${ }^{34}$. Indeed, the recent progress in the unfolding/(un)binding mechanisms of the various domains present in the dockerin/cohesin III complex (CBM and Xmod) may explain this rare event ${ }^{47,57}$. Different unbinding pathways have been reported for the unbinding of the dockerin/cohesin III complex due to unfolding of the Xmod domain, with a recognizable high unfolding force peak with a contour length of $\sim 34 \mathrm{~nm}$, before complete unbinding ${ }^{46}$. We also observed this behaviour in $47 \%$ of the successful curves (curves 3 and 6). In these cases, the final rupture forces were lower than those of one-step dissociation, as has been reported before, suggesting a structural stabilization function of Xmod ${ }^{28}$. Interestingly, we also observed two other dockerin/cohesin III unbinding signatures featuring an additional intermediate state before complete unbinding (curve 2) and before Xmod unfolding (curve 3), occurring in about $\sim 4 \%$ and $\sim 6 \%$ of the traces, respectively, leading to similar contour lengths of $12 \pm 4 \mathrm{~nm}$ and $10 \pm 5 \mathrm{~nm}$, respectively. These values are close to the one reported recently for type I dockerin $(\sim 8 \mathrm{~nm})$, interpreted as partial unbinding of the dockerin binding loop 1 and helix $1^{57}$. Our reported contour lengths correspond to the stretched length of 25-30 residues, consistent with the expected calcium binding loop and helix 1 of type III dockerin ${ }^{58}$. Thus, it is possible, that the step preceding titin unfolding mentioned above was due to this partial dockerin III detachment but occurring at lower forces. A detailed interpretation of dockerin III intermediate states would require site-directed mutagenesis, linker insertion and/or combination with molecular dynamics simulations, which is beyond the scope of this work. Thus, care should be taken when studying intermediate unfolding of the POI using the dockerin/cohesin III approach. Thus, the dockerin/cohesin III approach may not be the most appropriate method to assess intermediate states. However, we expect that the continuously evolving understanding of the unfolding and (un)binding mechanisms of the dockerin/cohesin family will make it an optimal system for robust and systematic study of protein unfolding ${ }^{47,57}$.

The CBM domain of the cohesin III molecule reveals a relatively weak conformation unfolding at forces $120 \pm 20 \mathrm{pN}$. Interestingly, despite the very low probability of titin I91 unfolding before the CBM domain (Fig. S2), these events were also observed (curves 4 and 
5). According to the forced unfolding probability, the expected rate of these events was of $\sim 2 \%$, similar the $2.8 \%$ that we observed experimentally. This obviously reflects the stochastic nature of the unfolding process and further validates the proposed approach as a tool to observe rare and low populated events.

With a relatively higher probability than all the other rare events, traces presenting less than eight titin 191 domains were also detected. This could be due to unsuccessful expression or misfolding of individual titin 191 repeats. However, in most of the traces, the extension at unbinding was in agreement with the expected $\sim 385 \mathrm{~nm}$ of the full molecule, suggesting that unfolding at low forces may happen due to incomplete refolding between curves (curve 6). Actually, the probability of observing traces with missing titin domains was higher when probing continuously a same spot of the surface $(72 \%$ of all traces presenting missing unfolding events) than when probing a fresh spot (28\%, Fig. S3). This suggests that the same molecule was probed repeatedly and, thus, some domains did not have time to completely refold in the time between force curves ( $\sim 3$ seconds) ${ }^{21,59}$. Partial titin denaturation and attachment to the surface may also explain missing titin unfolding events, as reflect the early, unspecific peaks in curve 6 . Nevertheless, $\sim 28 \%$ of the traces with less than eight titin unfolding events showed a total distance shorter than the expected contour length (trace 7). Given the relatively long folded length of the $\mathrm{T}_{8}$-dockerin III molecule $(45 \mathrm{~nm})$, this might be due to unspecific attachment of molecules to the surface via one of the titin domains. Although highly improbable (given the high unbinding forces of dockerin/cohesin III), this unfolding phenotype might also result from the dissociation of the dockerin/cohesin III complex before unfolding of the eight-titin domains. This interpretation may suggest an alternative mechanically more stable conformation of titin, that would sustain forces much higher than the common folded state. Finally, and occurring only once, a trace showing up to 12 titin unfolding peaks was also observed (Fig. S4). This may reflect uncontrolled oligomerization of $\mathrm{T}_{8}$ molecules during expression and purification.

\section{Multiple unfolding events}

Although the cantilever tip apex is relatively sharp, the tip apex is typically functionalized with multiple cohesin III complexes. Therefore, unfolding of multiple complexes is observed if the sample surface is densely populated with the molecular construct. Therefore, a rather sparse surface functionalization is recommended to minimize multiple binding. The surface density of molecules may be tuned by the incubation time of Sfp ligation reactions and/or proper protein concentration. During our experiments, simultaneous pulling of multiple $\mathrm{T}_{8}$ molecules was sometimes observed. While the mechanical response of multiple bonds in 
parallel has been addressed in the literature ${ }^{6,18,60-66}$, simultaneous unfolding of multiple molecules has been often regarded as a drawback. The use of site-specific strategies using high functionalization densities opens the door to study the mechanical response multiple molecules pulled in parallel, which may represent a more physiologically relevant configuration for some systems, like titin ${ }^{48,67}$. We observed several curves interpretable as multiple events, featuring generally higher unfolding forces and shorter separation between peaks than single event curves. While multiple events with more than two molecules were rare and difficult to interpret, double events were clearly discernible. Thus, we focused our analysis on double events only. Representative examples of double pulling traces are shown in Fig. 5. We analysed the unfolding events of curves only with clear double binding events revealing an average rupture force of $270 \pm 60 \mathrm{pN}($ mean $\pm \mathrm{SD})$, about $100 \mathrm{pN}$ higher than the average single domain unfolding forces and twice as spread (Fig, 5B). Like in the case of single molecule pulling, early peaks tend to have lower forces than latter ones, ranging from $\sim 230 \mathrm{pN}$ to $370 \mathrm{pN}$. The larger uncertainty in the last two points reflect poorer statistics as some of the curves showed less than 16 titin peaks due to simultaneous unfolding. Interestingly, the contour lengths followed a bimodal distribution, with a wide peak centred at $\sim 13 \mathrm{~nm}$ and a sharper peak centred at $\sim 28 \mathrm{~nm}$, the expected contour length of I91 (Fig. 5c), the latter corresponding to $\sim 35 \%$ of the unfolding events and suggesting simultaneous unfolding of two domains. Thus, the domains of two molecules pulled simultaneously tend to unfold either sequentially with a contour length difference between peaks of around half of the contour length of one domain ( $\sim 14 \mathrm{~nm}$ for 191) or simultaneously ( $\sim 28 \mathrm{~nm})$. Sequential unfolding led to a wide peak of forces at $\sim 260 \mathrm{pN}$, about $100 \mathrm{pN}$ higher than single domain unfolding. The force, and thus loading rate, shared between the two molecules that unfold alternatively. Simultaneous unfolding revealed, instead, a bimodal force distribution with peaks at $\sim 250 \mathrm{pN}$ and $310 \mathrm{pN}$ centred at $\sim 28 \mathrm{~nm}$ contour length. The high force peak, with a slightly lower value than twice the unfolding force of the single domain, is consistent with available models of parallel unbinding of multiple bonds, which predict a force of $\sim 330 \mathrm{pN}^{6}$, $60,68,69$. The lower force peak, however, reflects a more complex behaviour that would require detailed analysis. Regardless of the unfolding configuration, the forces are systematically and importantly higher than those required to unfold a single domain. This may be physiologically relevant, as titin molecules are loaded in parallel in muscles, implying that the forces required to unfold individual domains are importantly higher if multiple molecules are loaded in parallel. Thus scaling of single molecule data to the muscle should be done with caution. 


\section{Conclusions}

In force spectroscopy measurements, the probability of observing a single molecule unfolding/unbinding event is relatively low. Thus, a high number of approach-retract cycles is required. In addition to the low probability, grabbing the molecules from the desired specific locations is also largely improbable without specific pulling, resulting in an unknown attachment position and uncontrolled probing direction. More importantly, unspecific unfolding/unbinding forces may be recorded compromising the reliability of the obtained results. Furthermore, the use of unspecific methods require strict selection criteria that may lead to rejection of traces presenting important, although unexpected unfolding/unbinding mechanisms i.e. rare events. Therefore, robust techniques for protein immobilization are crucial to improve binding efficiency, reproducibility and to obtain large statistics in force unfolding experiments. Site-specific attachment provides the required robustness. Here, we show that probing the POI via the mechanically strong cohesin/dockerin III complex improves the specificity of force measurements and enhances the probability of grabbing the molecule from specific sites, providing precise knowledge of the pulling direction, higher efficiency and larger statistics. Moreover, the dockerin/cohesin III approach provides a robust and valuable tool to assess the unfolding history on multi-domain molecules with unambiguous determination of the number of pulled domains and provides large statistics to unravel rare events. Finally, it allows probing unfolding of multiple proteins pulled in parallel. We expect that the discovery of new mechanically stable receptor-ligand complexes will allow even more robust, reproducible and controlled unfolding measurements on an even larger number of proteins ${ }^{70}$.

\section{Supplementary Material}

Supplementary figures S1- S4.

\section{Acknowledgements}

This work was supported by Agence National de la Recherche grants BioHSFS ANR-15CE11-0007. A.M. was supported by a Long Term EMBO Fellowship (ALTF 1427-2014) and a Marie Curie Action (MSCA-IF-2014-EF-655157). We thank to Michael Nash and Wolfgang Ott for sharing the XMod-dockerin-III and cohesin-III plasmids and for technical assistance, and to Hirohide Takahashi and Renaud Vincentelli for technical assistance on cloning, expression and purification of the fusion proteins. 


\section{Figure Captions}
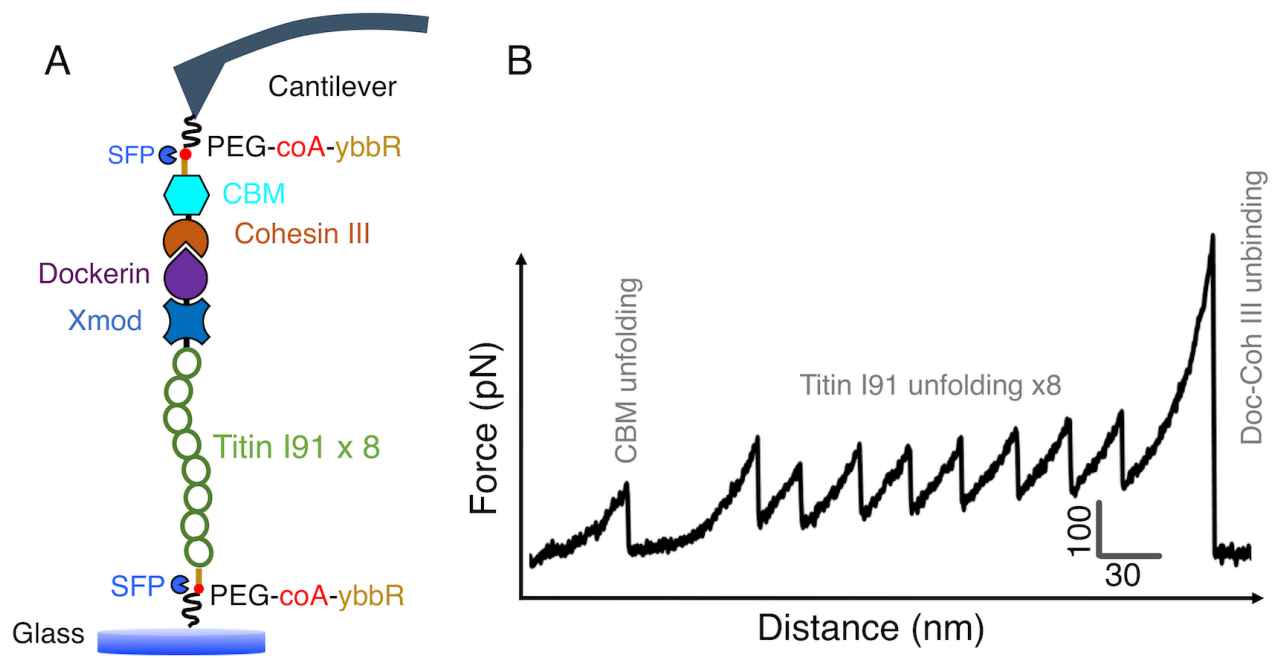

Figure 1. Experimental design. (A) Schematic representation of the experimental setup showing the site-specific pulling configuration. (B) A force-distance trace showing complete unfolding of covalently anchored (titin I91)x8 and Xmod-dockerin to the sample surface probed by covalently anchored cohesin III-CBM on the tip. The signature curve shows the unfolding of single CBM domain followed by unfolding of 8 titin I91 domains and then the dissociation of dockerin-cohesin III complex.

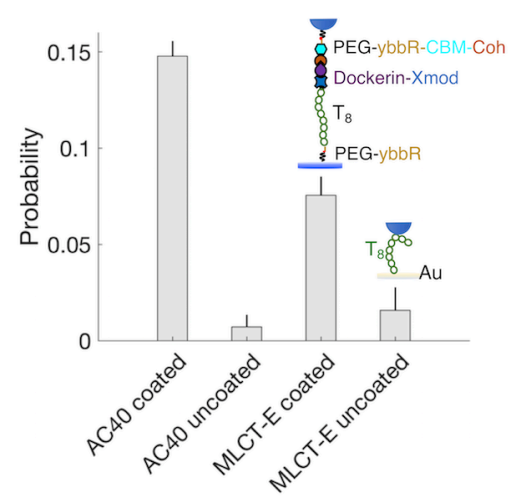

Figure 2. Probability of observing complete unfolding traces of a single molecular construct, without any unexpected unfolding regime, with the different cantilevers and coating conditions. Error bars show the standard deviation. 


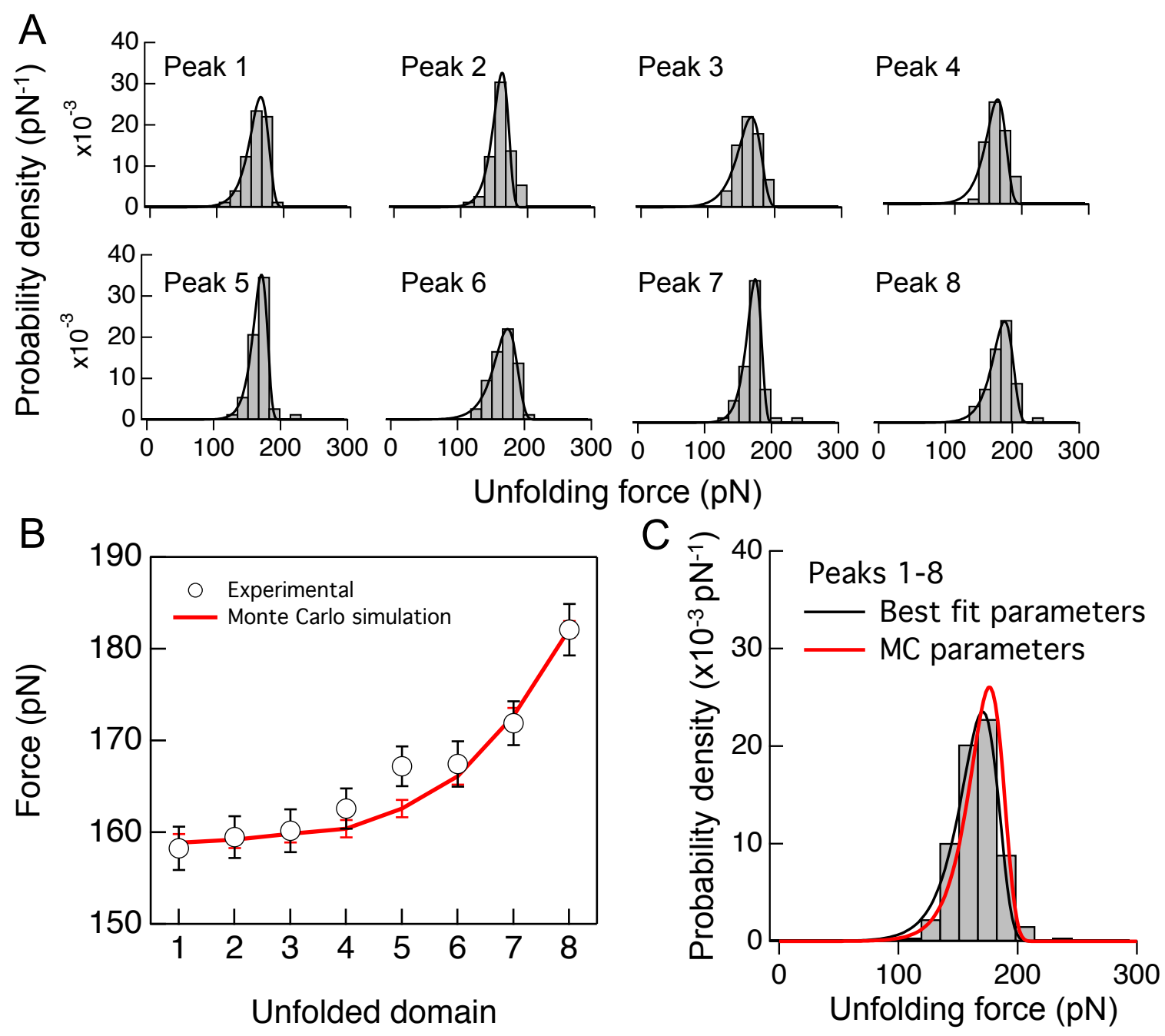

Figure 3. Unfolding history of titin domains. A) Probability density of unfolding forces for the eight titin domains calculated from $\sim 50$ events per plot. B) Average unfolding forces for each titin domain obtained from the plots in A). The red solid line show Monte Carlo simulations of eight titin unfolding domains with parameters $k_{\mathrm{u}}{ }^{0}=0.001 \mathrm{~s}^{-1}$ and $x_{\mathrm{u}}=0.29 \mathrm{~nm}$. Symbols are mean \pm standard error of the mean. C) Probability density of unfolding forces obstained pooling all peaks together. The solid black line represents the best fit with parameters $\left(k^{0}=4.4 \times 10^{-3} \mathrm{~s}^{-1}\right.$ and $\left.x_{\mathrm{u}}=0.26 \mathrm{~nm}\right)$. The solid red line is the predicted distribution using the parameters in B. 


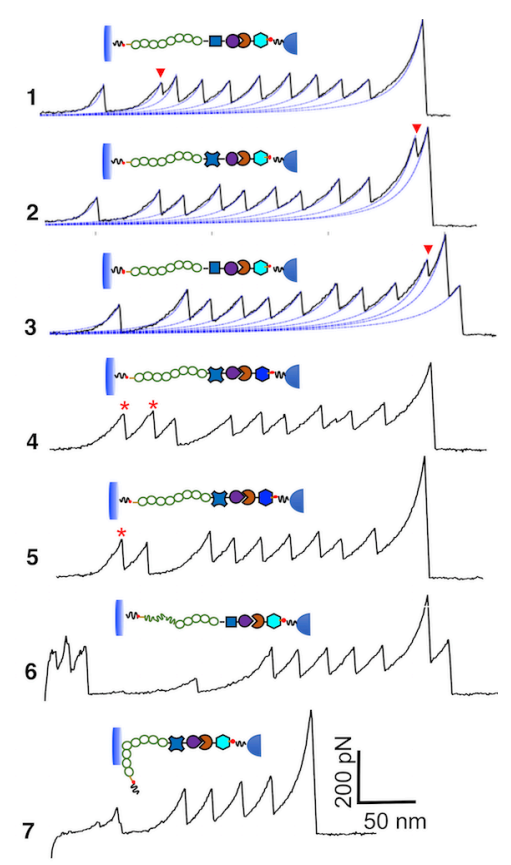

Figure 4. Examples of rare events observed during unfolding. Intermediate unfolding with a contour length of 10-13,5 nm before titin unfolding (trace 1, $L_{\mathrm{C}}=13,5 \mathrm{~nm}$ ), before Doc-Coh III unbinding (trace 2, $L_{\mathrm{C}}=10 \mathrm{~nm}$ ) and Xmod unfolding (trace $3, L_{\mathrm{C}}=11,1 \mathrm{~nm}$ ) are labelled with red arrows. The dockerin III having this rare partial unfolding step is indicated as magenta circle in the corresponding cartoon of the traces 1-3. Unfolding of Xmod domain prior to dissociation of Doc-Coh III complex (traces 3 and 6) where the Xmod is shown as a blue square in the corresponding cartoons. Traces 4 and 5 show the unlikely unfolding of titin I91 domains before the weaker CBM domain (traces 4 and 5). Titin I91 domains unfolded before $\mathrm{CBM}$ are labelled in red asterisks and illustrated as dark blue hexagon in the corresponding cartoons. Some traces showed less than eight titin I91 unfolding peaks dissociating at the expected, full length (trace 6) and at shorter lengths (trace 7). The missing titin domains are indicated as green springs in the corresponding cartoon illustration of trace 6 and the unexpected surface anchoring of titin I91 is depicted in the corresponding cartoon of trace 7. Unfolding traces with unexpected titin I91 unfolding events as in (traces 1 and 4-7) were excluded for analysis of the unfolding history (Fig. 3). 
A
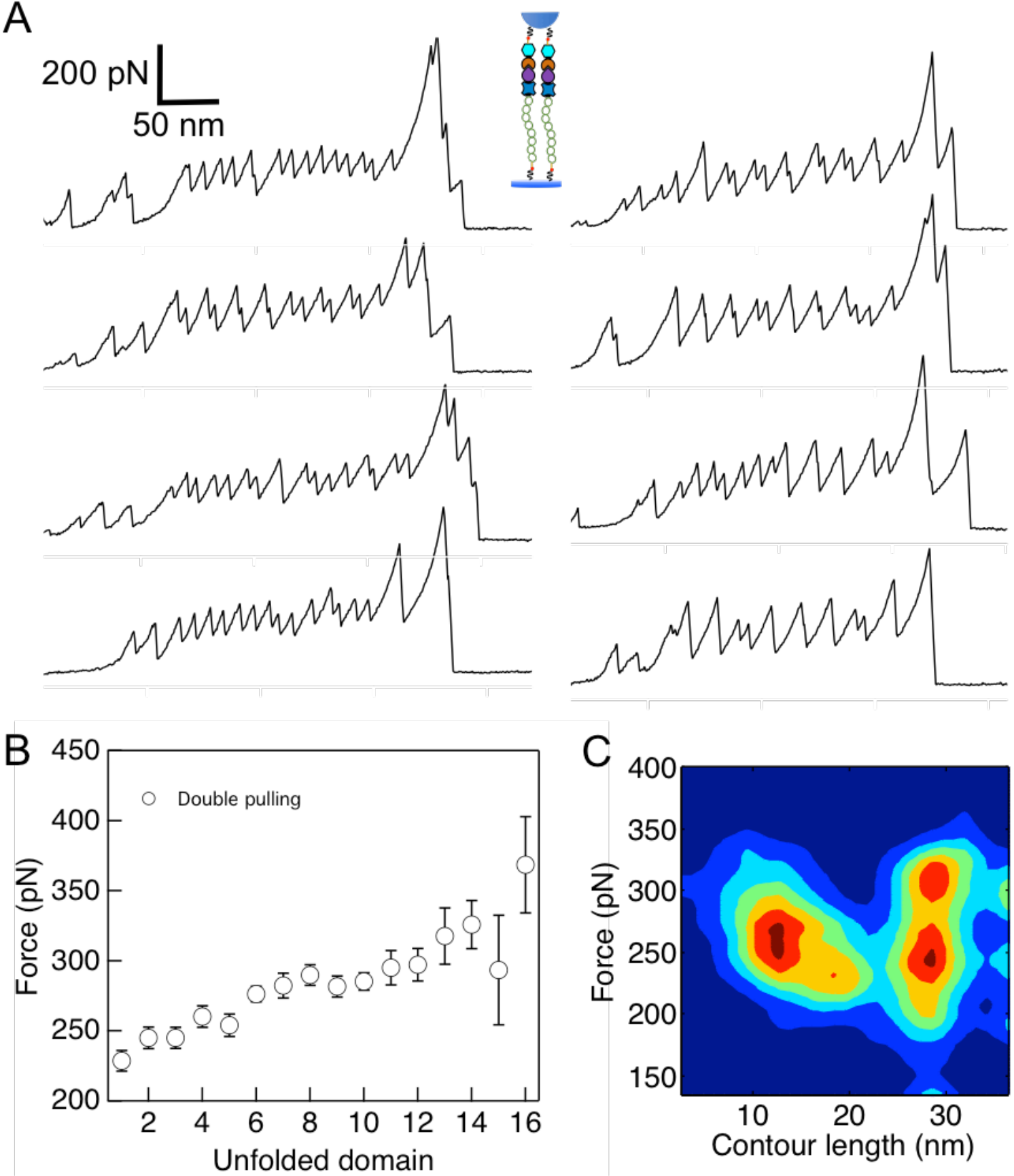

Figure 5. Simultaneous unfolding of multiple molecules. A) Examples of simultaneous pulling of two $T_{8}$ molecules revealing sequential (short separation between peaks) and simultaneous unfolding evens (long separation). B) Average unfolding forces for each titin I91 domains obtained from double pulling configuration. Error bars indicate the standard error of the mean. C) Contour plot of unfolding forces versus contour length from events showing simultaneous pulling of two molecules (data obtained from over 400 unfolding events. Colour scale represents the number of events). 
1. L. Tskhovrebova, J. Trinick, J. A. Sleep and R. M. Simmons, Nature 387 (6630), 308312 (1997).

2. $\quad$ E. L. Florin, V. T. Moy and H. E. Gaub, Science 264 (5157), 415-417 (1994).

3. V. T. Moy, E. L. Florin and H. E. Gaub, Science 266 (5183), 257-259 (1994).

4. M. Rief, M. Gautel, F. Oesterhelt, J. M. Fernandez and H. E. Gaub, Science 276 (5315), 1109-1112 (1997).

5. P. E. Marszalek, H. Lu, H. Li, M. Carrion-Vazquez, A. F. Oberhauser, K. Schulten and J. M. Fernandez, Nature 402 (6757), 100-103 (1999).

6. P. M. Williams, Anal. Chim. Acta 479 (1), 107-115 (2003).

7. O. K. Dudko, Quarterly Reviews of Biophysics 49 (2015).

8. M. Hinczewski, C. Hyeon and D. Thirumalai, Proceedings of the National Academy of Sciences 113 (27), E3852-E3861 (2016).

9. M. Carrion-Vazquez, A. F. Oberhauser, S. B. Fowler, P. E. Marszalek, S. E. Broedel, J. Clarke and J. M. Fernandez, Proceedings of the National Academy of Sciences 96 (7), 3694-3699 (1999).

10. F. Rico, L. Gonzalez, I. Casuso, M. Puig-Vidal and S. Scheuring, Science 342 (6159), 741-743 (2013).

11. F. Kong, Z. Li, William M. Parks, David W. Dumbauld, Andrés J. García, A. P. Mould, Martin J. Humphries and C. Zhu, Molecular Cell 49 (6), 1060-1068 (2013).

12. V. I. Zarnitsyna, J. Huang, F. Zhang, Y.-H. Chien, D. Leckband and C. Zhu, Proceedings of the National Academy of Sciences 104 (46), 18037-18042 (2007).

13. B. T. Marshall, K. K. Sarangapani, J. Lou, R. P. McEver and C. Zhu, Biophysical journal 88 (2), 1458-1466 (2005).

14. R. C. Zinober, D. J. Brockwell, G. S. Beddard, A. W. Blake, P. D. Olmsted, S. E. Radford and D. A. Smith, Protein science 11 (12), 2759-2765 (2002).

15. K. M. Tych, M. L. Hughes, J. Bourke, Y. Taniguchi, M. Kawakami, D. J. Brockwell and L. Dougan, Physical Review E 91 (1), 012710 (2015).

16. D. E. Makarov, Biophysical journal 96 (6), 2160-2167 (2009).

17. D. E. Makarov, P. K. Hansma and H. Metiu, The Journal of Chemical Physics 114 (21), 9663-9673 (2001).

18. R. B. Best, D. J. Brockwell, J. L. Toca-Herrera, A. W. Blake, D. A. Smith, S. E. Radford and J. Clarke, Analytica Chimica Acta 479 (1), 87-105 (2003).

19. D. J. Brockwell, G. S. Beddard, J. Clarkson, R. C. Zinober, A. W. Blake, J. Trinick, P. D. Olmsted, D. A. Smith and S. E. Radford, Biophysical journal 83 (1), 458-472 (2002).

20. I. Schwaiger, A. Kardinal, M. Schleicher, A. A. Noegel and M. Rief, Nat Struct Mol Biol 11 (1), 81-85 (2004).

21. A. F. Oberhauser, P. E. Marszalek, M. Carrion-Vazquez and J. M. Fernandez, Nature Structural \& Molecular Biology 6 (11), 1025-1028 (1999).

22. P. Hinterdorfer, W. Baumgartner, H. J. Gruber, K. Schilcher and H. Schindler, Proc. Natl. Acad. Sci. U. S. A. 93 (8), 3477-3481 (1996).

23. X. H. Zhang, E. Wojcikiewicz and V. T. Moy, Biophys. J. 83 (4), 2270-2279 (2002).

24. D. A. Pippig, F. Baumann, M. Strackharn, D. Aschenbrenner and H. E. Gaub, ACS nano 8 (7), 6551-6555 (2014).

25. I. Popa, R. Berkovich, J. Alegre-Cebollada, C. L. Badilla, J. A. s. Rivas-Pardo, Y. Taniguchi, M. Kawakami and J. M. Fernandez, Journal of the American Chemical Society 135 (34), 12762-12771 (2013).

26. B. Zakeri, J. O. Fierer, E. Celik, E. C. Chittock, U. Schwarz-Linek, V. T. Moy and M. Howarth, Proceedings of the National Academy of Sciences 109 (12), E690-E697 (2012).

27. M. Otten, W. Ott, M. A. Jobst, L. F. Milles, T. Verdorfer, D. A. Pippig, M. A. Nash and H. E. Gaub, Nature methods 11 (11), 1127-1130 (2014). 
28. C. Schoeler, K. H. Malinowska, R. C. Bernardi, L. F. Milles, M. A. Jobst, E. Durner, W. Ott, D. B. Fried, E. A. Bayer and K. Schulten, Nature Communications 5 (2014).

29. E. Durner, W. Ott, M. A. Nash and H. E. Gaub, ACS Omega 2 (6), 3064-3069 (2017).

30. K. C. Neuman, T. Lionnet and J. F. Allemand, Annual Review of Materials Research 37 (1), 33-67 (2007).

31. E. Celik and V. T. Moy, Journal of Molecular Recognition 25 (1), 53-56 (2011).

32. Y. Taniguchi and M. Kawakami, Langmuir 26 (13), 10433-10436 (2010).

33. R. Walder, M.-A. LeBlanc, W. J. Van Patten, D. T. Edwards, J. A. Greenberg, A. Adhikari, S. R. Okoniewski, R. M. A. Sullan, D. Rabuka, M. C. Sousa and T. T. Perkins, Journal of the American Chemical Society 139 (29), 9867-9875 (2017).

34. S. W. Stahl, M. A. Nash, D. B. Fried, M. Slutzki, Y. Barak, E. A. Bayer and H. E. Gaub, Proceedings of the National Academy of Sciences 109 (50), 20431-20436 (2012).

35. J. Yin, P. D. Straight, S. M. McLoughlin, Z. Zhou, A. J. Lin, D. E. Golan, N. L. Kelleher, R. Kolter and C. T. Walsh, Proceedings of the National Academy of Sciences of the United States of America 102 (44), 15815-15820 (2005).

36. J. L. Zimmermann, T. Nicolaus, G. Neuert and K. Blank, nature protocols 5 (6), 975985 (2010).

37. M. L. Hughes and L. Dougan, Reports on Progress in Physics 79 (7), 076601 (2016).

38. J. Yin, A. J. Lin, D. E. Golan and C. T. Walsh, Nature Protocols 1 (1), 280 (2006).

39. J. E. Sader, J. A. Sanelli, B. D. Adamson, J. P. Monty, X. Wei, S. A. Crawford, J. R. Friend, I. Marusic, P. Mulvaney and E. J. Bieske, Review of Scientific Instruments 83 (10), 103705 (2012).

40. J. E. Sader, R. Borgani, C. T. Gibson, D. B. Haviland, M. J. Higgins, J. I. Kilpatrick, J. Lu, P. Mulvaney, C. J. Shearer, A. D. Slattery, P.-A. Thorén, J. Tran, H. Zhang, H. Zhang and T. Zheng, Review of Scientific Instruments 87 (9), 093711 (2016).

41. J. L. Hutter and J. Bechhoefer, Rev. Sci. Instrum. 64 (7), 1868-1873 (1993).

42. H. J. Butt and M. Jaschke, Nanotechnology 6, 1-7 (1995).

43. M. J. Higgins, R. Proksch, J. E. Sader, M. Polcik, S. Mc Endoo, J. P. Cleveland and S. P. Jarvis, Review of Scientific Instruments 77 (1), 013701 (2006).

44. H. Schillers, C. Rianna, J. Schäpe, T. Luque, H. Doschke, M. Wälte, J. J. Uriarte, N. Campillo, G. P. A. Michanetzis, J. Bobrowska, A. Dumitru, E. T. Herruzo, S. Bovio, P. Parot, M. Galluzzi, A. Podestà, L. Puricelli, S. Scheuring, Y. Missirlis, R. Garcia, M. Odorico, J.-M. Teulon, F. Lafont, M. Lekka, F. Rico, A. Rigato, J.-L. Pellequer, H. Oberleithner, D. Navajas and M. Radmacher, Scientific Reports 7 (1), 5117 (2017).

45. M. Rief, J. M. Fernandez and H. E. Gaub, Physical Review Letters 81 (21), 4764-4767 (1998).

46. C. Schoeler, K. H. Malinowska, R. C. Bernardi, L. F. Milles, M. A. Jobst, E. Durner, W. Ott, D. B. Fried, E. A. Bayer, K. Schulten, H. E. Gaub and M. A. Nash, Nat Commun 5 (2014).

47. T. Verdorfer, R. Bernardi, A. Meinhold, W. Ott, Z. Luthey-Schulten, M. A. Nash and H. E. Gaub, Journal of the American Chemical Society (2017).

48. H. Li, W. A. Linke, A. F. Oberhauser, M. Carrion-Vazquez, J. G. Kerkvliet, H. Lu, P. E. Marszalek and J. M. Fernandez, Nature 418 (6901), 998-1002 (2002).

49. B. I. Costescu, S. Sturm and F. Gräter, Journal of Structural Biology (2016).

50. Y. Cao, R. Kuske and H. Li, Biophysical journal 95 (2), 782-788 (2008).

51. P. M. Williams, S. B. Fowler, R. B. Best, J. Luis Toca-Herrera, K. A. Scott, A. Steward and J. Clarke, Nature 422 (6930), 446-449 (2003).

52. A. F. Oberhauser, P. K. Hansma, M. Carrion-Vazquez and J. M. Fernandez, Proceedings of the National Academy of Sciences of the United States of America 98 (2), 468-472 (2001). 
53. R. B. Best, S. B. Fowler, J. L. Toca-Herrera and J. Clarke, Proceedings of the National Academy of Sciences 99 (19), 12143-12148 (2002).

54. P. Hanggi, P. Talkner and M. Borkovec, Reviews of Modern Physics 62 (2), 251-341 (1990).

55. T.-L. Kuo, S. Garcia-Manyes, J. Li, I. Barel, H. Lu, B. J. Berne, M. Urbakh, J. Klafter and J. M. Fernández, Proceedings of the National Academy of Sciences 107 (25), 1133611340 (2010).

56. S. Garcia-Manyes, Bruji, J. cacute, C. L. Badilla and J. M. Fernández, Biophysical journal 93 (7), 2436-2446 (2007).

57. M. A. Jobst, L. F. Milles, C. Schoeler, W. Ott, D. B. Fried, E. A. Bayer, H. E. Gaub and M. A. Nash, Elife 4, e10319 (2015).

58. O. Salama-Alber, M. K. Jobby, S. Chitayat, S. P. Smith, B. A. White, L. J. Shimon, R. Lamed, F. Frolow and E. A. Bayer, Journal of Biological Chemistry 288 (23), 16827-16838 (2013).

59. H. Chen, G. Yuan, R. S. Winardhi, M. Yao, I. Popa, J. M. Fernandez and J. Yan, Journal of the American Chemical Society 137 (10), 3540-3546 (2015).

60. T. A. Sulchek, R. W. Friddle, K. Langry, E. Y. Lau, H. Albrecht, T. V. Ratto, S. J. DeNardo, M. E. Colvin and A. Noy, Proc. Natl. Acad. Sci. U. S. A. 102 (46), 16638-16643 (2005).

61. T. V. Ratto, R. E. Rudd, K. C. Langry, R. L. Balhorn and M. W. McElfresh, Langmuir 22 (4), 1749-1757 (2006).

62. C. Rankl, F. Kienberger, L. Wildling, J. Wruss, H. J. Gruber, D. Blaas and P. Hinterdorfer, Proceedings of the National Academy of Sciences of the United States of America 105 (46), 17778-17783 (2008).

63. S. Guo, C. Ray, A. Kirkpatrick, N. Lad and B. B. Akhremitchev, Biophysical Journal 95 (8), 3964-3976 (2008).

64. J.-M. Teulon, Y. Delcuze, M. Odorico, S.-w. W. Chen, P. Parot and J.-L. Pellequer, Journal of Molecular Recognition 24 (3), 490-502 (2011).

65. C. Chu, E. Celik, F. Rico and V. T. Moy, PLoS ONE 8 (5), e64187 (2013).

66. S. Guo, N. Li, N. Lad, S. Desai and B. B. Akhremitchev, The Journal of Physical Chemistry C 114 (19), 8755-8765 (2010).

67. W. Linke, Histology and histopathology 15 (3), 799-812 (2000).

68. E. Evans, Annu. Rev. Biophys. Biomol. Struct. 30, 105-128 (2001).

69. T. Sulchek, R. W. Friddle and A. Noy, Biophys J 90 (12), 4686-4691 (2006).

70. P. Herman, S. El-Kirat-Chatel, A. Beaussart, J. A. Geoghegan, T. J. Foster and Y. F. Dufrêne, Molecular microbiology 93 (2), 356-368 (2014). 